\title{
Non-contrast MRI Findings of Adhesive Capsulitis: A review
}

\author{
Aida Karami ${ }^{1}$, Parviz Ghezelbash ${ }^{1}$, Mohammad Qorbanisani ${ }^{1}$, Zahra Ghezelbash ${ }^{1}$, Amir Hossein Norooznezhad ${ }^{2}$, \\ Parisa Karami ${ }^{1^{*}}$
}

${ }^{1}$ Department of Radiology, School of Medicine, Zanjan University of Medical Sciences, Zanjan, Iran. ${ }^{2}$ Medical Biology Research Center, Technology Health Research Institute, Kermanshah University of Medical Sciences, Kermanshah, Iran.

Adhesive Capsulitis (AC) is a self-limiting disease of the shoulder joint characterized by progressive painful restriction of the shoulder's motion. This study aimed to search the findings on AC in non-contrast MRI. Although AC is a clinical diagnosis, imaging can provide helpful data for earlier diagnosis and treatment. It seems that some findings are specific and sensitive for accurate diagnosis and staging of $\mathrm{AC}$ such as coracohumeral ligament thickening, joint capsule edema, and rotator interval infiltration. Non-contrast MRI can provide an abundance of information on AC and help clinicians make a more definitive diagnosis, stage the disease, and choose better treatment plans.

Keywords: adhesive capsulitis, frozen shoulder, periarthritis, magnetic resonance imaging, bursitis, inflammation, diagnosis

\section{Introduction}

The term adhesive capsulitis (AC) was first used by Neviaser to explain findings such as chronic inflammation in the capsule of the shoulder joint resulting in adherence of periarthritis soft tissue [1]. Many studies on the imaging of AC exist, most of them based on MR arthrography or contrast enhanced MRI [2-4]. These techniques are somewhat invasive with specific protocols. Thus, some researchers have tried to describe imaging findings in the routine MRI which are more readily available. The current research attempted to review studies accordingly and describe the most sensitive and specific routine, noncontrast MRI findings in AC. Thus, any article on MR arthrography or contrast enhanced MRI findings of the disease, except those pointing to non-contrast MRI findings in $\mathrm{AC}$ patients was excluded from the study.

\section{MRI findings}

Major imaging findings on AC are based on pathologic changes in the disease. Synovitis, and the thickening and contracting of the glenohumeral joint capsule are considered major microscopic pathologic findings of $\mathrm{AC}$ and, theoretically, are detectable in imaging. Such changes are also responsible for the patients' clinical signs and symptoms [5].
In 2008, Sofka et al. noted that there were no reports in the literature on specific non-contrast MRI findings that correspond with the clinical stages of AC. Thus, they tried to explain the correlation between $\mathrm{AC}$ imaging findings and clinical staging using non-contrast MRI [6]. They studied 46 patients with a clinical diagnosis of adhesive capsulitis focusing on the pathological, morphological, and signal changes of the joint capsule as well as the presence of scarring in the rotator cuff interval. Moreover, they tried to find a relationship between capsular thickening and hyperintensity on MRI with capsular hypertrophy and hypervascular synovitis as described in different pathologic stages of the disease.

They concluded that capsule-synovial thickening (measured at the midaxillary pouch) shows the greatest correlation with clinical staging. According to their results, greater combined synovial and capsular thickening is detectable in early stages of the disease. However, in the late fibrotic stages, only capsular thickening may be seen. They also assessed capsular signals in proton density (PD) fat saturation MR images and compared them with normal anterior capsule signals. This led them to the conclusion that capsular signal hyperintensity is more related to stage two of the disease. In addition, they considered rotator interval scarring to be a non-specific sign of AC not correlated with clinical stages.

Personal non-commercial use only. Rheumatology Research Journal. Copyright (C 2020. All rights reserved

*Corresponding Author: Parisa Karami, M.D., Rheumatology Research Center (RRC), Zanjan MRI Center, Vali-e-Asr Square, Zanjan, Iran. PO-Box: 4515777978, E-mail: parisakarami1363@yahoo.com, Telefax: +98-243-378-3729.

Received: 03 December 2019; Accepted: 27 January 2020 
Shortening and thickening of the coracohumeral ligament have been described as another characteristic finding of $\mathrm{AC}$ in some studies. The coracohumeral ligament is seen as a homogeneous low signal linear structure at sagittal T1-W MR images [4, 7-10].

In 2011, Jin-Qing Li et al. studied the MRIs of 72 shoulders of patients with a clinical diagnosis of AC and compared them with 120 healthy controls. They showed that the resultant thickening of the coracohumeral ligament in patients may be a useful sign for the diagnosis of $\mathrm{AC}$ [11]. They measured the diameter of the coracohumeral ligament in sagittal oblique $\mathrm{T} 1-\mathrm{W}$ images and showed that it was significantly thicker in patients with AC (3.99 \pm 1.68 $\mathrm{mm})$ than in the control group $(3.08 \pm 1.32 \mathrm{~mm})$. Interestingly, they also found that the coracohumeral ligament cannot be visualized in some normal people. The researchers stated that the visualization of this ligament as a hyposignal structure was facilitated by fatty tissue surrounding it in the rotator cuff interval. They further mentioned that the lack of visualization may be due to the absence of fatty tissue in some of the normal population. On the other hand, the CHL visualization rate is significantly lower in patients with $\mathrm{AC}$ than in the normal population, regarding obliteration of this space with fibrosis in the course of the disease; therefore, no visualization of the coracohumeral ligament might be a potential restriction for using this sign. Jin-Qing Li et al. also found that obliteration of the subcoracoid fat triangle is a useful yet non-specific sign for AC.

Another conventional MRI finding was described by Gondim Teixeira et al., in 2012. They studied 34 MRIs of shoulders in patients with AC [12]. They noticed that capsular changes are the most common findings in different phases of $\mathrm{AC}$; therefore, capsular signal change and thickening could be useful diagnostic signs. However, they also noticed that when using the conventional MRI protocol, limitation in shoulder distension in comparison to MR arthrogram is a potential drawback for the accurate measurement of capsular thickness. Therefore, they studied inferior glenohumeral ligament (IGHL) signal changes in patients with a clinical diagnosis of AC. IGHL is a capsular thickened area contiguous with the inferior aspect of the joint capsule. The researchers used T2 fat saturation MR images in the oblique coronal plane and compared the IGHL signal with the long head of the biceps tendon and noticed a specific (88.2\%) and sensitive (85.5$88.2 \%)$ sign: hyperintensity of the inferior glenohumeral ligament in adhesive capsulitis. They also introduced the extracapsular hyperintense layer on T2 WI as a new sign of AC.

In 2012, Zhao et al. published a study on findings of adhesive capsulitis in Asian symptomatic patients. They studied 60 people with a clinical diagnosis of AC and tried to describe the relationship between the pathologic changes of the rotator interval (as a critical involved area in this disease) and imaging findings of the disease [13]. They evaluated the coracohumeral ligament, articular capsule thickening, and fatty space under the coracoid process using oblique sagittal T1 and found that partial or complete obliteration of subcoracoid fat and thickening of the CHL and capsule at the rotator interval in comparison with the control group (4.2 vs. $2.4 \mathrm{~mm}, 7.2$ vs. $4.4 \mathrm{~mm}$, respectively; $p$-value $<0.05)$ are characteristic findings for AC. They also mentioned that bicipital tendon synovitis could be considered as a specific abnormality in AC.

In 2015, Shungon Park et al. tried to connect adhesive capsulitis MRI and clinical findings [14]. They studied 103 patients with AC and compared their severity of pain, range of motion, and clinical stage with MRI findings. They described extracapsular edema as a useful sign, which is consistent with an area of signal change bordering the outer capsular surface that Teixeira et al. reported [12] and showed the correlation between these findings and clinical limited range of motion. They also pointed out that capsular edema is the most common MRI finding in AC associated with loss of motion range on external rotation. Mean capsular thickness of the humeral portion of the axillary recess was about $4.06 \mathrm{~mm}$ in AC patients in this study, and it was found to be associated with the patients' pain levels. Joint capsule edema and obliteration of the subcoracoid fat triangle were significantly more common in the early stages of AC [14].

In another study published in 2017, Chi A.S. et al. investigated non-contrast MRI findings in patients with clinical AC [15]. They studied 31 patients and evaluated coracohumeral ligament thickness, axillary recess thickening or edema, and rotator interval infiltration and calculated the sensitivity and specificity of diagnosis based on using one, two, or all three of these criteria. They also conjoined the level of clinical suspicion with MR findings criterion for an accurate diagnosis.

The researchers concluded that coracohumeral ligament thickening is $76.7 \%$ sensitive and $53.3 \%$ specific for the diagnosis of AC. explained that lower sensitivity of coracohumeral ligament thickness in previous studies is related to higher thickness cut-off; thus, they considered 2 $\mathrm{mm}$ coracohumeral ligament thickness for a diagnosis of AC. They also used this finding alone in high clinical suspicion settings.

The specificity and sensitivity of coracohumeral ligament thickening and rotator interval infiltration as two criteria were $55.2 \%$ and $66.7 \%$, respectively. Using these findings in moderate clinical suspicion settings could be helpful because of the higher specificity rate.

Sensitivity and specificity for coracohumeral ligament thickening, axillary recess thickening/edema, and rotator interval infiltration as three criteria were $23.3 \%$ and $86.7 \%$, respectively. Regarding the highest specificity rate with using all of these three criteria, it would be helpful in the setting of low clinical suspicion or atypical signs of adhesive capsulitis. Chi et al. concluded that non-contrast MRI shoulder examinations can be diagnostic for adhesive capsulitis without direct MR arthrography.

Studies that included calculated sensitivity and specificity of investigated variables for the diagnosis of adhesive capsulitis are provided in Table 1. 
Table 1. Sensitivity and specificity of magnetic resonance imaging (MRI) findings for diagnosis of adhesive capsulitis.

\begin{tabular}{|c|c|c|c|c|}
\hline Study & Contrast & Finding(s) & Sensitivity & Specificity \\
\hline \multirow{3}{*}{$\begin{array}{c}\text { Chi et al } \\
(2017)\end{array}$} & No & Coracohumeral ligament thickening $(>2 \mathrm{~mm})$ & $76.7 \%$ & $53.3 \%$ \\
\hline & No & Coracohumeral ligament thickening + Rotator interval infiltration & $66.7 \%$ & $55.2 \%$ \\
\hline & No & $\begin{array}{c}\text { CHL thickening }+ \text { Rotator interval infiltration }+ \text { Axillary recess } \\
\text { edema/pericapsular edema }\end{array}$ & $23.3 \%$ & $86.7 \%$ \\
\hline \multirow{5}{*}{$\begin{array}{c}\text { Gondim } \\
\text { Teixeira } \\
\text { et al } \\
(2012)\end{array}$} & No & Inferior glenohumeral ligament T2 WI hyperintensity & $\begin{array}{l}85.3- \\
88.2 \%\end{array}$ & $88.2 \%$ \\
\hline & No & Subcoracoid fat triangle obliteration & $\begin{array}{c}41.2- \\
50.0 \%\end{array}$ & $\begin{array}{l}67.6- \\
73.5 \%\end{array}$ \\
\hline & No & Coracohumeral ligament thickness ( $>4 \mathrm{~mm}, \mathrm{~T} 1 \mathrm{WI}$ sagittal) & $\begin{array}{l}21.8- \\
31.0 \%\end{array}$ & $\begin{array}{l}89.2- \\
93.3 \%\end{array}$ \\
\hline & Yes & Rotator interval enhancement & $\begin{array}{l}47.0- \\
61.8 \%\end{array}$ & $\begin{array}{l}76.5- \\
94.1 \%\end{array}$ \\
\hline & Yes & Inferior glenohumeral ligament enhancement & $\begin{array}{l}82.3- \\
94.0 \%\end{array}$ & $88.2 \%$ \\
\hline
\end{tabular}

\section{Discussion}

Adhesive capsulitis (AC), also known as frozen shoulder, is a self-limiting condition first described in 1896 by Duplay as a case report [16]. In 1934, Codman described "frozen shoulder" as a painful range of motionreducing phenomenon of a shoulder joint [17]. As previously mentioned, "adhesive capsulitis" was first used by Neviaser to explain findings such as chronic inflammation in the capsule of the shoulder joint resulting in adherence of periarthritis soft tissue [1].

Patients affected with AC typically present with progressive painful restriction in the range of passive and active motions of the shoulder joint. Although the etiology of idiopathic $\mathrm{AC}$ is still unknown, there are several risk factors associated with this condition such as diabetes mellitus (DM), older age, female gender, hypertension, dyslipidemia, and thyroid dysfunction [18, 19]. Frozen shoulder is commonly seen between the ages of 35 and 60 [20].

The clinical course of AC has been described in 4 stages. The first stage is defined by the presence of shoulder pain for almost 1 to 3 months and normal or mild limited, painful active and passive motions. In the second stage, the patients' symptoms include chronic pain in both active and passive motions and limitations in abduction, internal and external rotation, and forward flexion lasting for almost 3 to 9 months. In the third stage of the disease, symptom duration (significant loss of motion and minimal pain except at the end of range of motion) is about 9 to 15 months. In the fourth stage, the patient has had symptoms for 15 to 24 months during which the patient has less pain and the range of motion improves [21].

These clinical stages are concomitant with the pathologic changes of the disease. Inflammatory changes lead to ligamentous thickening and contraction which progress into fibrosis causing adherence of periarthritis soft tissue. These changes affect the rotator interval space and present imaging changes which can be seen in magnetic resonance imaging (MRI). Nevertheless, AC is a clinical diagnosis for which imaging will be useful under certain circumstances, especially when the patient presents atypical symptoms or is a candidate for an invasive procedure [22].

Although there are many articles on contrast MRI and MR arthrographic findings, both of these procedures are somewhat invasive and therefore not suitable for patients in terms of cost and benefit. There are few studies on the role of non-contrast MRI in helping with AC diagnosis or staging.

As the alternate name for this disease (adhesive periarthritis) describes, inflammatory changes lead to edema, which progresses into fibrosis. Fibrotic changes in joint surrounding structures lead to capsular and ligamentous stiffness.

It is noteworthy that all of the studies performed on non-contrast MRI findings agree with capsular changes in the progression of shoulder adhesive capsulitis; however, this research compared those studies to find better MRI sequences and the best methods for evaluating these diagnostic points.

Sofka et al. used oblique coronal and axial PD sequences to evaluate capsulosynovial changes at the inferior part of the midaxillary pouch [6]; Zhao et al., on the other hand, noticed that most changes occur at the rotator cuff interval. They used oblique sagittal T1 WI to evaluate the capsule at the rotator cuff interval [13]. Both of these studies concluded that thickening of the capsule is a characteristic finding for $\mathrm{AC}$. 
Teixeira et al. also confirmed capsular changes in the progression of AC [12]. However, they noticed the difficulty of capsular measurements in some patients in contracting the capsule. Therefore, they advised using oblique coronal $\mathrm{T} 2$ fat sat sequences for a better evaluation of $\mathrm{AC}$ for findings of signal changes in the inferior recess of the joint capsule (IGHL). Previously, Sofka et al. mentioned that capsular signal changes in PD fat sat sequences occur in the second stage of the disease [6]. Park et al. also found a relationship between capsular edema and loss of motion range [14].

As previously mentioned, involvement of the rotator cuff interval has been described in this disease. This triangular space is located between the supraspinatus and subscapularis tendons and CHL. CHL forms the roof of this space covering the superior glenohumeral ligament (SGHL) and the bicipital tendon near the medial border of this space. CHL thickening, measured at oblique sagittal T1 WI by Li et al. [11] and Zhao et al. [13] (3.9 mm and $4.2 \mathrm{~mm}$, respectively), has been described as a characteristic finding in AC. However, as previously mentioned, this criterion sometimes is not useful as it does not visualize CHL in a certain fraction of the general population. Li et al. advised using fat suppressed PD images to find the high signal soft tissue in the rotator cuff interval as a useful finding in these patients [11]. There are some differences in the lower limit of normal CHL thickness which influence the sensitivity of this finding. Chi et al. mentioned that a CHL thickness greater than 2 $\mathrm{mm}$ is a sensitive finding which could be used in high clinical suspicion settings [15].

Obliteration of subcoracoid fat as a characteristic finding was mentioned by Zhao et al. [13]. They described it in oblique sagittal T1 W images. Rotator interval infiltration was mentioned by $\mathrm{Li}$ et al. as a nonspecific finding [11]. Li et al. also mentioned infiltration of rotator cuff fat and described it in PD fat suppressed sequences when CHL could not be evaluated [11]. Moreover, rotator interval infiltration was used by Chi et al. to increase the specificity of imaging criteria in patients with equivocal symptoms in addition to capsular and CHL thickening [15].

It seems that more studies are necessary to confirm the accuracy of other findings like bicipital synovitis, the abnormality mentioned by Zhao et al. [13], or the T2 hyperintense extracapsular layer described by Teixeira et al. [12].

\section{Conclusion}

According to the articles on non-contrast MRI findings in patients with $\mathrm{AC}$ that were reviewed in this study, there are findings that can be specific, sensitive, and helpful to the diagnosis or staging of $\mathrm{AC}$, such as coracohumeral ligament (CHL) thickening, capsular thickening or edema, and rotator interval infiltration.

It seems that non-contrast MRI can provide much information about adhesive capsulitis and help clinicians in making more definitive diagnoses, staging the disease course, and choosing better treatment plans. Some noncontrast MRI findings are sensitive and specific enough to be used in clinics. However, more studies should be planned to more precisely determine the role of noncontrast MRI in the diagnosis and staging of adhesive capsulitis of the shoulder.

\section{References}

1. Neviaser JS. Adhesive capsulitis of the shoulder: a study of the pathological findings in periarthritis of the shoulder. J Bone Joint Surg 1945; 27(2):211-22.

2. Jung J-Y, Jee W-H, Chun HJ, Kim Y-S, Chung YG, Kim J-M. Adhesive capsulitis of the shoulder: evaluation with MR arthrography. Eur Radiol 2006; 16(4):791-96. doi: 10.1007/s00330-005-0020-5.

3. Lefevre-Colau M-M, Drapé J-L, Fayad F, Rannou F, Diche T, Minvielle F. et al. Magnetic resonance imaging of shoulders with idiopathic adhesive capsulitis: reliability of measures. Eur Radiol 2005; 15(12):2415-22. doi: 10.1007/s00330-005-2830-x.

4. Mengiardi B, Pfirrmann CW, Gerber C, Hodler J, Zanetti M. Frozen shoulder: MR arthrographic findings. Radiology 2004; 233(2):486-92. doi: 10.1148/radiol.2332031219.

5. Lundberg BJ. The Frozen shoulder: clinical and radiographical observations the effect of manipulation under general anesthesia structure and glycosaminoglycan content of the joint capsule local bone metabolism. Acta Orthop Scand suppl 1969; 40(sup119):1-59. doi: 10.3109/ort.1969.40.suppl119.01 .

6. Sofka CM, Ciavarra GA, Hannafin JA, Cordasco FA, Potter HG. Magnetic resonance imaging of adhesive capsulitis: correlation with clinical staging. HSS $\boldsymbol{J}$ 2008; 4(2):164-69. doi: 10.1007/s11420-008-9088-1.

7. Omari A, Bunker TD. Open surgical release for frozen shoulder: surgical findings and results of the release. J Shoulder Elbow Surg 2001; 10(4):353-57. doi: $10.1067 / \mathrm{mse} .2001 .115986$

8. Ozaki J, Nakagawa Y, Sakurai G, Tamai S. Recalcitrant chronic adhesive capsulitis of the 
shoulder. Role of contracture of the coracohumeral ligament and rotator interval in pathogenesis and treatment. J Bone Joint Surg Am 1989; 71(10):151115 .

9. NEER CS, Satterlee CC, Dalsey RM, Flatow EL. The anatomy and potential effects of contracture of the coracohumeral ligament. Clin Orthop Relat Res 1992; 280:182-85.

10. Hand G, Athanasou N, Matthews T, Carr A. The pathology of frozen shoulder. J Bone Joint Surg Br 2007; 89(7):928-32. doi: 10.1302/0301620X.89B7.19097

11. Li J-q, Tang K-1, Wang J, Li Q-y, Xu H-t, Yang H-f. et al. MRI findings for frozen shoulder evaluation: is the thickness of the coracohumeral ligament a valuable diagnostic tool? PLoS One 2011; 6(12):e28704. doi: 10.1371/journal.pone.0028704

12. Teixeira PAG, Balaj C, Chanson A, Lecocq S, Louis M, Blum A. Adhesive capsulitis of the shoulder: value of inferior glenohumeral ligament signal changes on T2-weighted fat-saturated images. Am J Roentgenol 2012; 198(6):W589-96. doi: 10.2214/AJR.11.7453

13. Zhao W, Zheng X, Liu Y, Yang W, Amirbekian V, Diaz LE. et al. An MRI study of symptomatic adhesive capsulitis. PLoS One 2012; 7(10):e47277. doi: 10.1371/journal.pone.0047277

14. Park S, Lee D-H, Yoon S-H, Lee HY, Kwack K-S. Evaluation of adhesive capsulitis of the shoulder with fat-suppressed T2-weighted MRI: association between clinical features and MRI findings. $\boldsymbol{A m} \boldsymbol{J}$ Roentgenol 2016; 207(1):135-41. doi: 10.2214/AJR.15.15200
15. Chi AS, Kim J, Long SS, Morrison WB, Zoga AC. Non-contrast MRI diagnosis of adhesive capsulitis of the shoulder. Clin Imaging 2017; 44:46-50. doi: 10.1016/j.clinimag.2017.04.002

16. Ott O, Hertel S, Gaipl U, Frey B, Schmidt M, Fietkau R. Benign painful shoulder syndrome: initial results of a single-center prospective randomized radiotherapy dose-optimization trial. Strahlenther Onkol 2012; 188(12):1108-13. doi: 10.1007/s00066-012-0237-6

17. Codman E. Codman E: The Shoulder: Rupture of the supraspinatus tendon and other lesions in or about the subacromial bursa. Boston, Massachuset, Krieger Publishing; 1934.

18. Reeves B. The natural history of the frozen shoulder syndrome. Scand J Rheumatol 1975;4(4):193-96. doi: 10.3109/03009747509165255.

19. Cakir M, Samanci N, Balci N, Balci MK. Musculoskeletal manifestations in patients with thyroid disease. Clin Endocrinol 2003; 59(2):162-67. doi: $10.1046 /$ j.1365-2265.2003.01786.x

20. Hsu JE, Anakwenze OA, Warrender WJ, Abboud JA. Current review of adhesive capsulitis. J Shoulder Elbow Surg 2011; 20(3):502-14. doi: 10.1016/j.jse.2010.08.023.

21. Hannafin JA, Chiaia TA. Adhesive Capsulitis: A Treatment Approach. Clin Orthop Relat Res 2000; (372):95-09.

22. Sano H, Hatori M, Mineta M, Hosaka M, Itoi E. Tumors masked as frozen shoulders: a retrospective analysis. J Shoulder Elbow Surg 2010; 19(2):262-66. doi: 10.1016/j.jse.2009.05.010. 
